\title{
Analysis of ocular hemodynamic using combined STdOCT and ultrasonic methods
}

\author{
M.E. Danielewska, ${ }^{* 1}$ D. Szlag, ${ }^{2}$ D.R. Iskander ${ }^{3}$ and M. Wojtkowski ${ }^{2}$ \\ ${ }^{1}$ Institute of Physics, Wroclaw University of Technology, Wybrzeże Wyspiańskiego 27, 50-370 Wroclaw, Poland \\ ${ }^{2}$ Institute of Physics, Nicolaus Copernicus University, Grudziadzka 5, 87-100 Torun, Poland \\ ${ }^{3}$ Institute of Biomedical Engineering and Instrumentation, Wroclaw University of Technology, \\ Wybrzeże Wyspiańskiego 27, 50-370 Wroclaw, Poland
}

Received November 15, 2011; accepted December 13, 2011; published December 31, 2011

\begin{abstract}
A new approach to the human eye hemodynamic assessment and analysis is presented. A combined method of ultrasonic ocular pulse measurements and registration of retinal blood flow velocity using STdOCT synchronically with heart activity was applied. Spectral analysis and coherence analysis were used to characterize frequency components of considered signals. The fundamental frequency related to the heart rate present in the ECG signal was also noticed in the blood pulsation, longitudinal corneal apex displacements, and the ocular pulse spectrum derived from the STdOCT measurements. The proposed technique of new, noninvasive ocular circulation measurements and analysis might be helpful in diagnosing vascular ocular diseases such as, for example, normal tension glaucoma.
\end{abstract}

Investigating human eye hemodynamic is important in diagnosing many eye vascular diseases, particularly glaucoma [1]. Nowadays, increased intraocular pressure (IOP) is not the only crucial risk to develop glaucoma. Normal tension glaucoma (NTG) is a very common form of this disease. However, the aetiology of NTG has been considered as unknown. Blood circulation disorders inside the eye, as well as those in the cardiovascular system may play a significant role in the disease progression leading to the optic nerve damage.

Pulsatile ocular blood flow and IOP variations are the fundamental components of the phenomenon, known as the ocular pulse [2]. The expansion of the corneal and scleral surface is mainly caused by the ocular pulse, which depends also on heart activity [3,4]. The results obtained by Evans showed that amplitudes of the ocular pulse for healthy and glaucomatous patients, calculated using spectral analysis, are different [5]. Therefore, examining the relationships between the ocular pulse and cardiovascular activity might bring information about blood propagation to the eye globe.

Elementary methods of ocular blood flow measurements, used by many ophthalmologists, are invasive (fluorescein angiography) or limited by poor resolution (laser Doppler flowmetry) [6,7]. On the other hand, the methods of in-vivo retinal blood flow

*E-mail: monika.danielewska@pwr.wroc.pl measurements, which are based on the optical Doppler effect combined with time [8-10] or Fourier domain optical coherence tomography (OCT) [11-12], are limited by low acquisition rate or measurement time. One of the most promising methods to measure and image ocular blood flow is joint spectral and time domain Optical Coherence Tomography (STdOCT) [13]. This technique offers high sensitivity preserved in acquisition speed over 50000 lines/s [14, 15].

Of interest is also accurate measure of longitudinal corneal apex displacements (LCAD). LCAD is a purely mechanical response to blood pressure and ocular circulation which was found to be well correlated with cardiopulmonary signals. Hence, simultaneous measurements of LCAD with retinal blood velocity might be helpful to assess the influence of ocular blood flow on ocular pulse amplitude.

The main goal of this study was to combine the following methods: (i) noninvasive measurement of the ocular pulse with ultrasound sensors on one eye, (ii) registration of blood flow velocity with STdOCT in the fellow eye, and (iii) measurement of heart activity.

LCAD of the right eye was measured noninvasively using an ultrasonic transducer, functioning at a frequency of $0.8 \mathrm{MHz}$. The technical details of this method were described earlier [16]. The retinal vessels of the left eye were registered using a typical SOCT setup. All crosssectional images were measured in the same position with a small lateral range. The blood flow velocity in a selected retinal vessel was retrieved using STdOCT method with high transverse oversampling for establishing accurate data of blood flow velocity and at a reasonable high frame rate to examine in-vivo retinal hemodynamic. Involuntary movements of object introduce offset in flow velocities. These artifacts were removed using the STdOCT bulk motion correction algorithm described in [17]. SOCT data were directly synchronized with a pulseoximeter (one Ascan corresponds to one point in the pulse plot). A schematic diagram of the measurement setup is presented in Fig. 1. 
Synchronically with the signal of retinal blood flow (RBF), the following signals of heart activity were recorded: electrocardiogram (ECG) and pulse wave using pulseoximeter, placed on the right earlobe. Measurements were carried out five times for two healthy volunteers aged 27 and 29, who did not exhibit any eye and heart pathologies. LCAD signal was sampled at a frequency of $100 \mathrm{~Hz}$, while the remaining signals at $66 \mathrm{kHz}$.

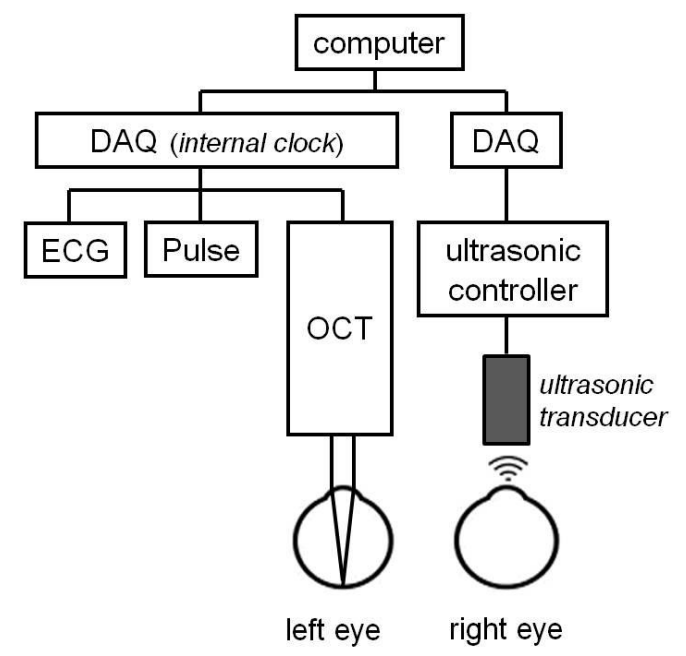

Fig. 1. The concept of measurements the ocular pulse (right eye) with ultrasonic transducer, retinal blood flow using OCT (left eye) synchronically with ECG and pulse signals.

Obtained data were numerically processed. First, the signals were detrended by removing a linear trend. Then, a band-pass filter $(0.1-20 \mathrm{~Hz})$ was applied to remove frequencies unrelated to heart activity.

LCAD and heart activity related signals were considered before [18, 19]. To compare the signal of blood flow velocity registered with STdOCT to the signals of LCAD and cardiac activity, a technique to extract RBF velocity was applied. This consisted of fitting a 2D Gaussian function with parameters $\left(\mathrm{A}, \mu_{\mathrm{x}}, \mu_{\mathrm{y}}, \sigma_{\mathrm{x}}, \sigma_{\mathrm{y}}\right.$, $\delta)$ to an image formed by concatenation of $5000 \mathrm{~A}$-scans. The parameter A describes the background around the measured vessel, while $\left(\mu_{\mathrm{x}}, \mu_{\mathrm{y}}\right),\left(\sigma_{\mathrm{x}}, \sigma_{\mathrm{y}}\right)$ and $\delta$ describe the center, spread and azimuth of the Gaussian fit, respectively. Subsequently, the volume enclosed by the Gaussian fit $\left( \pm 3 \sigma_{x} ; \pm 3 \sigma_{y}\right)$ was used as an estimator (arbitrary units) of retinal blood flow.

Finally, a Fourier Transform was applied to calculate spectral characteristics for all signals. To obtain information about the phase compliance between corresponding frequency components contained in two given signals, the coherence function was evaluated. The coherence function is bounded between 0 and 1 and indicates synergy between the two signals. The coherence of zero indicates that two given signal components at a certain frequency are independent while, if the coherence value is one, they are perfectly correlated (i.e., their phases change in time in the same way) [20].

In this study we present the results obtained for subject I (27 years old woman, emmetrope). The time and spectral characteristics of all registered signals are shown in Fig. 2 and Fig. 3, respectively.

In all signals presented in Fig. 2, the pulsative character of their time representations is observed. Moreover, they are nonstationary [18], meaning that their frequency components vary in time. The main factor which influences these phenomena is heart rate variability.
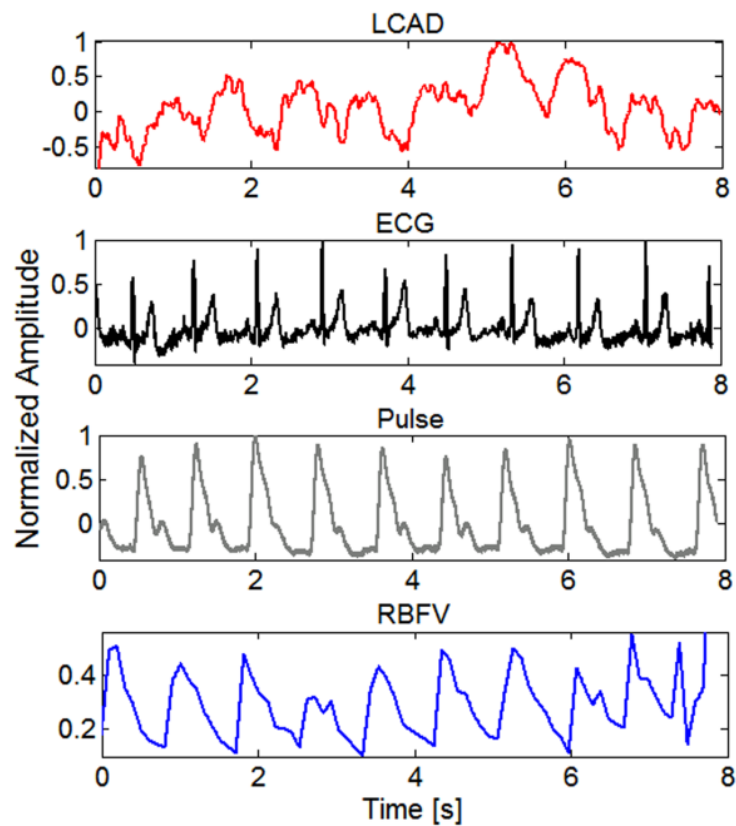

Fig. 2. Time representation of registered signals: longitudinal corneal apex displacements (LCAD), ECG signal, blood pulsation (Pulse) and retinal blood flow velocity (RBFV).
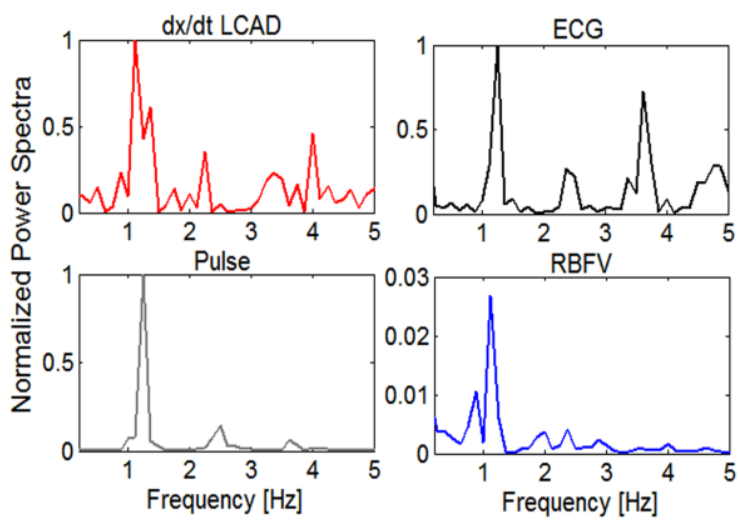

Fig. 3. Normalized power spectra of considered signals.

In Fig. 3 power spectrum of longitudinal corneal apex velocity (first derivative of the position in time ) is shown, to visualize the spectral characteristic of LCAD, which decays quite fast for higher frequencies. According to the 
properties of a Fourier Transform, the derivative of the function is proportional to the product of the Fourier Transform of this function and the argument of Fourier Transformation (frequency). Therefore, in the presented case higher harmonics are pronounced in an LCAD signal derivative.

In all spectra (Fig. 3) the frequency which corresponds to the heart rate (about $1.2 \mathrm{~Hz}$ ) can be observed. Frequency components of pulse and RBFV signals appear to be more similar in comparison to the spectra of LCAD and the ECG signal.

The coherence function values calculated between LCAD and RBFV (Fig. 4), as well as between Pulse and RBFV indicate moderate correlation between the signals at the first harmonic of the heart rate, i.e., around $2.4 \mathrm{~Hz}$.

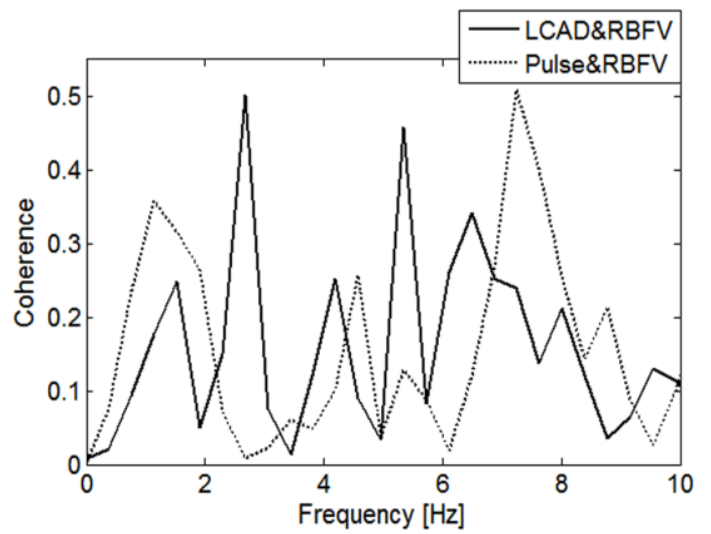

Fig. 4. The coherence function between particular pairs of signals.

The proposed method combining ultrasonic measurements of LCAD and STdOCT measurement of RBFV helps to simultaneously observe pulsatile blood flow in retinal vessels, ocular pulse response, and corneal displacements. It was noticed that spectral components of the acquired signals, measured with ultrasonic and STdOCT methods, reveal certain similarities. The fundamental frequencies related to the heart rate and its first harmonic were visible in all spectra. However, the coherence between the LCAD and RBFV signals or between pulse and RBFV signals for that frequency was found to be moderate (up to $50 \%$ ). This indicates that amplitude spectral characteristic of the considered signals cannot be viewed alone [5], and that techniques focusing on phase relationships between those signals also need to be considered [19].

Noninvasive measurement of the longitudinal eye displacements and retinal blood flow signals and investigation of their spectral correlation with cardiac activity could lead to a better understanding of blood circulation on the way from heart to eye. The proposed technique might be a promising tool for ocular hemodynamic assessment and diagnostic of ocular vascular diseases.

It is worth noting that the proposed method is limited to healthy subjects without any oculomotor pathology (i.e., nystagmus). Plans are made to utilise the presented method to measure subjects with ocular and cardiovascular pathologies (e.g., diabetic retinopathy, macular degeneration or retinal artery and vein occlusion). Also, it is of interest to compare dynamics in two eyes but the current setup prohibits such studies.

Part of this work was supported by funds from The Foundation for Polish Science (project VENTURES/2011-7/4 to M.E.D.). Daniel Szlag and Maciej Wojtkowski acknowledge EUROHORCS, ESF, and the Foundation for Polish Science for support within the frames of European Young Investigator Award (EURYI - 01/2008-PL).

We would like to thank Małgorzata Kowalska for help in the experimental phase of this project.

\section{References}

[1] J. Flammer, S. Orgul, V.P. Costa, N. Orzalesi, G.K. Krieglstein, L.M. Serra, J.P.Renard, E. Stefansson, Prog. Retin. Eye Res. 21, 359 (2002).

[2] R.B. Northrop, S.S. Nilakhe, IEEE Trans. Biomed. Eng. BME 24, 139 (1977).

[3] D. Trew, C. James, S. Thomas, R. Sutton, S. Smith, Graefes. Arch. Clin. Exp. Ophthalmol. 229,553 (1991).

[4] T. Bosley, M. Cohen, W. Gee, J. Reed III, 24, 6 Stroke (1993).

[5] D.W. Evans, S.L. Hosking, S.J. Embleton, A.J. Morgan, J.D. Barlett, Graefes Arch. Clin. Exp. Ophthalmol. 240, 475 (2002).

[6] L.A. Yannuzzi, K.T. Rohrer, L.J. Tindel, R.S. Sobel, M.A. Costanza, W. Shields, E. Zang, Ophthalmology 93, 611 (1986).

[7] T.H. Williamson, A. Harris, Br. J. Ophtahalmol. 78, 939 (1994).

[8] S. Yazdanafar, A.M. Rollins, J.A. Izatt, Opt. Lett. 25, 1448 (2000).

[9] A.M. Rollins, S. Yazdanafar, J.K. Barton, J.A. Izatt, J. Biomed. Opt. 7(1), 123 (2002).

[10] V. Westpfal, S. Yazdanafar, A.M. Rollins, J.A. Izatt, Opt. Lett. 27, 34 (2002).

[11] R.A. Leitgeb, L. Schmetterer, W. Drexler, A.F. Fercher, R.J. Zawadzki, T. Bajraszewki, Opt. Exp. 11, 3116 (2003).

[12] B.R. White, M.C. Pierce, N. Nassif, B. Cense, B. Hyle Park, G.J. Tearney, B.E. Bouma, Opt. Exp. 11, 3490 (2003).

[13] M. Wojtkowski, Appl. Opt. 49, D30 (2010).

[14] M. Szkulmowski, A. Szkulmowska, T. Bajraszewski, A. Kowalczyk, M. Wojtkowski, Opt. Exp. 16, 6008 (2008).

[15] M. Szkulmowski, I. Grulkowski, D. Szlag, A. Szkulmowska, A. Kowalczyk, M. Wojtkowski, Opt. Exp. 14, 14281 (2009).

[16] M.A. Kowalska, H.T. Kasprzak, D.R. Iskander, M. Danielewska, D. Mas, IEEE Trans. Biomed. Eng. 58, 674 (2011).

[17] A. Szkulmowska, M. Szkulmowski, D. Szlag, A. Kowalczyk, M. Wojtkowski, Opt. Exp. 17, 10584 (2009).

[18] H. Kasprzak, D.R. Iskander, Eye 21, 1212 (2007).

[19] M.E. Danielewska, D.R. Iskander, M. Kowalska, H.T. Kasprzak, Eye (submitted 2011).

[20] A.S. Eadie, J.R. Pugh, B. Winn, Ophthalmic Physiol. Opt. 15, 311 (1995). 